\title{
NDA Via Gamma-Ray Active and Passive Computed Tomography
}

\author{
D. J. Decman \\ H. E. Martz \\ G. P. Roberson \\ E. Johansson
}

October 1996

This is an informal report intended primarily for internal or limited external distribution. The opinions and conclusions stated are those of the author and may or may not be those of the Laboratory.

Work performed under the auspices of the U.S. Department of Energy by the Lawrence Livermore National Laboratory under Contract W-7405-Eng-48. 


\section{DISCLAIMER}

This document was prepared as an account of work sponsored by an agency of the United States Government. Neither the United States Government nor the University of California nor any of their employees, makes any warranty, express or implied, or assumes any legal liability or responsibility for the accuracy, completeness, or usefulness of any information, apparatus, product, or process disclosed, or represents that its use would not infringe privately owned rights. Reference herein to any specific commercial product, process, or service by trade name, trademark, manufacturer, or otherwise, does not necessarily constitute or imply its endorsement, recommendation, or favoring by the United States Government or the University of California. The views and opinions of authors expressed herein do not necessarily state or reflect those of the United States Government or the University of California, and shall not be used for advertising or product endorsement purposes.

This report has been reproduced directly from the best available copy.

Available to DOE and DOE contractors from the Office of Scientific and Technical Information

P.O. Box 62, Oak Ridge, TN 37831

Prices available from (615) 576-8401, FTS 626-8401

Available to the public from the

National Technical Information Service

U.S. Department of Commerce

5285 Port Royal Rd.,

Springfield, VA 22161 


\title{
NDA Via Gamma-Ray \\ Active And Passive Computed Tomography
}

\author{
Daniel J. Decman, Harry E. Martz, G. Patrick Roberson, and Erik Johansson \\ Lawrence Livermore National Laboratory \\ P.O. Box 808, Mail Stop L-231 \\ Livermore, CA 94551, USA
}

\section{Introduction}

The purpose of LLNL's effort is to develop a nondestructive assay (NDA) technology that will identify and accurately quantify all detectable radioisotopes in closed containers of waste, regardless of their classification: low level, transuranic or mixed, which contains radioactivity and hazardous organic species. The scope of our effort is to complete the development of a non-invasive waste drum scanner that uses the principles of computed tomography and gamma-ray spectral analysis to identify and quantify, all detectable radioisotopes within closed waste containers. Once this and other technologies are developed [WES94], waste drums can be non destructively and accurately categorized to satisfy repository and regulatory guidelines prior to sub-surface burial.

\section{Summary}

Gamma-ray-based computed tomography (CT) requires that two different measurements be made on a closed waste container.[MAR92 and ROB94] When the results from these two measurements are combined, it becomes possible to identify and quantify all detectable gamma-ray emitting radioisotopes within a container. All measurements are made in a tomographic manner, i.e., the container is moved sequentially through wellknown and accurately reproducible translation, rotation, and elevation positions in order to obtain gamma-ray data that is reconstructed by computer into images that represent waste contents.[ROB94]

The two measurements modes are called active (A) and passive (P) CT. In the ACT mode, a collimated gamma-ray source external to the waste container emits multiple, mono-energetic gamma rays that pass through the container and are detected on the opposite side. The attenuated gamma-rays transmitted are measured as a function of both energy and position of the container. Thus, container contents are "mapped" via the measured amount of attenuation suffered at each gamma-ray energy. In effect, a three dimensional (3D) image of gamma-ray attenuation versus waste content is obtained.

In the PCT measurement mode, the external radioactive source is shuttered (turned-off), and the waste container, is moved through similar positions used for the ACT measurements. However, this time the radiation detectors record any gamma-rays emitted by radioactive sources on the inside of the waste container. Thus, internal radioactive content is "mapped" or 3D-imaged in the same tomographic manner as the attenuating matrix materials were in the ACT measurement mode. 
When the PCT results are combined with the ACT results, one is able to make an energy dependent attenuation correction that properly accounts for the attenuation the heterogeneous matrix materials have on any gamma rays emitted from internal radioactive sources.[MAR95] In effect, we properly correct externally detected gamma rays for attenuation caused by the waste matrix, regardless of where the internal gammaray sources were located or what materials the radiation passed through. The gamma-ray spectra are used to identify all measured radioisotopes present. The position information after reconstruction provides localization as a function of radioisotope. Thus, we obtain accurate and quantitative radioactivity values for every isotope detected within the wastes, and these results should be able to provide the necessary data to "certify" and categorize the wastes so they can be transported and disposed of legally and at the least expense.

\section{Significance and Use}

Most DOE sites that have handled uranium (U) and/or plutonium (Pu) over the past 50 years, will require the use of several technologies that will enable them to identify and quantify the radioactive content within waste containers such as 208-liter drums (55gallon) and other sizes.[AND91 and PDP95] Preferably, as many waste containers as possible will be assayed non invasively, because the analysis cost per opened container is prohibitively expensive. Possible categories for wastes containing radioactivity include high level (HLW), transuranic (TRU), low level (LLW), or mixed (M). DOE's major decontamination and decommissioning (D\&D) effort will also generate radioactive wastes that must be categorized as TRU, LLW, or mixed. Such wastes also exist within the nuclear power industry; thus, the computed tomography technology, once developed, may be utilized in these industrial sectors to properly categorize and dispose of their radioactive wastes.

\section{Waste Characteristics and Source}

We have made initial tests of the A\&PCT technology on TRU waste drums at the Lawrence Livermore National Laboratory (LLNL), Rocky Flats Environmental Technology Site (RFETS), and Idaho National Engineering Laboratory (INEL). At LLNL the 55-gallon drums contained smaller containers with solidified chemical wastes. The RFETS wastes were all low density combustible matrices. At INEL the wastes included lead-lined drums with combustibles and a very dense sludge drum. In all cases the plutonium loading of the drums was less than 20 grams of ${ }^{239} \mathrm{Pu}$.

\section{Theory}

In order to assay an unknown waste drum using gamma-ray active and passive CT three techniques are required. These techniques are (1) gamma-ray spectroscopy data acquisition and analysis; (2) active and passive image reconstruction; and (3) A\&PCT data acquisition. It is useful to describe briefly the principles and issues associated with each of these techniques to better understand the challenge of NDA of waste drums. 


\section{Gamma-ray spectroscopy for radioactive assay}

Gamma-ray spectroscopy with high-energy resolution germanium detectors has been successfully used as a quantitative radioactive assay method for many years. The highenergy resolution allows for radionuclide identification with high confidence. This resolution also increases the signal to noise for the measurement thereby enabling very precise peak areas (or intensities) to be extracted from very complicated spectra. For point sources there are several methods available to relate these peak areas to absolute assay values. However for many sample-detector geometries employed the point source assumption is not valid and it is difficult to relate peak areas to absolute intensities without calibration sources of the same energy and geometry. This problem arises because of the difficulty in calculating the detector solid angle for extended sources; this is made even more complicated when sample self-absorption is important. For sources that are roughly the same size as the detector there are methods that can relate the extended source efficiency to point source calibration data. However these methods become less reliable as the source dimensions become much larger than the detector. For the problem that we are discussing here, 208-1 drums with 0.2-l detectors, these methods do not apply. Moreover, these conventional gamma ray spectroscopy methods require the source to be uniformly distributed in a homogeneous attenuating matrix, real waste drums rarely meet these conditions.

Gamma-ray spectroscopy attempts have been tried to solve the waste drum assay problem by applying the LANL developed segmented gamma-ray spectrometry (SGS) technique. The SGS technique is DOE's current state-of-practice in measuring gamma-rays from contained radioactive wastes. The SGS technique measures spatially averaged gammaray intensities in 8 or 10 segments, i.e., vertical slices of the drum. The average matrix attenuation value for each slice is measured by the transmission of an external source. The attenuation value is used to correct each section's average passive gamma-ray emitted intensity. The A\&PCT technology that we are developing is a more refined technique that improves the imaging from 10 large segments to many small volume elements-voxels.

The A\&PCT methods allow the gamma-ray spectroscopy portion of the absolute assay problem to be broken down into a simpler analysis. The passive measurement localizes the activity of interest into small sized $(5 \mathrm{~cm}$ on a side) voxels. The absolute detector efficiency for each of these elements can be directly related to calibration measurements of point sources. The active measurement provides the data for the attenuation correction. The absolute assay can then be obtained by adding the activities in each of the volume elements of the reconstructed image. Also, most of the nuclides of interest emit gammarays of more than one energy, this allows an additional check on the image reconstruction and the absolute assay results. 


\section{A\&PCT image reconstruction}

While it seems simple enough to assay the total radioactivity within a large distributed volume, such as a nuclear waste container, by measuring the emitted radiation, the central difficulty is that an accurate absolute assay is impossible unless the measured radiation can be corrected for the self-attenuation within the source. This correction requires knowledge of the spatial distribution and density of both emitters and absorbers throughout the volume. An accurate assay will therefore necessarily involve a complete determination of the 3D structure of the source even though the original problem posed by the regulations requires only one number, the total radioactivity quoted as a $\mathrm{Pu}$ effective number, contained within the waste drum.

As is well known, the 3D structure of an object function (e.g. density distribution or emitting radioactive source) can be determined remotely or externally by computed tomography in exactly the same way that bone structure in medical patients is computed from x-ray images taken at all angles around the patient. It is easy to see that the complete set of projection images or shadows contains the same quantity of information as a description of the original object. However, instead of the object being described as a density in 3D space with Cartesian coordinates $x, y$, and $z$, the description is in terms of a projected density in a direction $\phi$, and two Cartesian coordinates, $x$ and $h$, perpendicular to that direction. Since a projection, a simple sum, is a linear function there is a simple inverse relationship, known as the Radon transform, between the set of projections and the original function or object. This assures that the original function can be recovered from its projections. In fact there is a Fourier transform relationship between the projections and the original object.

However, when it comes to imaging a physical object with some particular system, the simple Radon transform does not of course adequately describe the system. A detector collimated to receive radiation from a fine line through a source does not measure a simple projected density, but rather the photon counts decreased as the square of the distance between the emitter and the detector, $1 / \mathbf{R}^{2}$, and decreased exponentially by absorption along the line of sight. In addition, the collimation of high energy photons is normally done with beam stops rather than lenses, and the collimator will have a diverging acceptance angle with a width or area proportional to distance through the source. Lastly, the effects of finite resolution will degrade the accuracy if the source, the waste container, has objects of high density contrast, that is either emitters or absorbers with sharp boundaries which are not well resolved.

The non-linear effects in the system, in particular the $1 / R^{2}$ fall-off, the exponential attenuation, and the spatially varying collimator response imply that reconstruction or inversion will not be a linear operation. Although emission tomography is not a new idea, most current applications do not fully account for these non-linear effects and do not provide for accurate quantitative measurements. In particular, in medical imaging, reconstructions are generally done with an inverse Radon transform. This implies the 
assumptions that the internal attenuation is not too strong, and that the distance through the object, the patient, is not too great so that the divergence of the collimators and the $1 / R^{2}$ fall-off are also small. In medical imaging it is possible to achieve a reconstruction of sufficient qualitative accuracy to be clinically useful in diagnosis. However, these effects cannot be ignored in quantitative imaging.

In order to correct the emitted radiation for self-absorption we need to know the attenuation throughout the volume of the object. This can be done with transmission tomography also called active tomography in which we measure the attenuation of an external source of penetrating radiation, an x-ray or gamma-ray beam, as it passes through the object of interest. In general this is a simpler problem than emission tomography, and the differences are instructive. First, in transmission tomography, the source position is fixed so the $1 / \mathbf{R}^{2}$ dilution of the radiation is known. Second, since we wish to measure only the attenuation itself, we may take the logarithm of the attenuation to convert the problem to a linear transform. Third, we can generally achieve much higher resolution and better signal to noise with transmission tomography. The external source may be collimated to a fine point so that the final resolution depends only on the size of the detectors. For non-living objects such as waste containers, the photon flux level can generally be made quite high. Better resolution and higher signal to noise result in more accurate and more stable reconstructions.

Iterative methods for reconstruction generally proceed by successive minimization or maximization of an objective function. For example, given an accurate description of the system including the non-linear effects, minimizing the squared difference between the predicted and observed data will result in an accurate reconstruction. Several numerical algorithms exist for least squares minimization. All proceed by moving down the gradient of the objective function, the sum of squared differences, for example, by -steepest descent, by conjugate directions, or by successive projections. The last is termed ART for Algebraic Reconstruction Technique. Alternatively, one may consider the data obtained as the result of a stochastic process based on a Poisson probability distribution. For any given model of the source, there is a certain probability of actually obtaining the measured data. The source may then be reconstructed by varying the model to maximize the probability of obtaining the measured data. Such algorithms go by the name of Maximum Likelihood-Expectation Maximization (MLEM). All these techniques are valid provided the instrument has been well modeled and sufficient data has been obtained with sufficient signal to noise.

\section{A\&PCT data acquisition}

Active and Passive computed tomography uses a scanner based on nuclear-spectroscopybased detectors and electronics. They differ from the conventional CT scanners in that they discriminate between photons of different energies. The quantity that is reconstructed is the attenuation value for some volume element, or voxel, at location $x, y, z$ within the object. The voxel size and clarity are defined by scan and image reconstruction parameters. The reconstruction algorithms require line integrals, also called ray sums, for many ray paths through the object. It is useful to note that the CT ray sum acquired at a single energy is analogous to a simple gamma-ray transmission gauge. The results are thus a discrete quantitative measurement of the linear attenuation 
coefficient at each energy measured i.e., there has been no integration over the energy spectrum. Such scanners provide ACT images with pixels that represent the absolute measurement of attenuation at specific energies. The linear attenuation coefficient $(\mu)$ is the parameter determined by image reconstruction of the ACT measurements. For a waste drum, the attenuation due to the drum's contents, whether heterogeneous or homogeneous, is accurately measured in the third dimension at different $z$ planes (or elevations) of the drum. Further, the ratio of attenuation images at two or more energies can be used to determine density and effective-atomic-number images. This may provide useful information for characterizing hazardous waste. The theory behind multiple, mono-energetic ACT data to determine effective atomic number is given elsewhere. [1]

Note that ACT does not measure the presence or identity of any radioisotope, or any source strength or activity within a waste drum. The function that is imaged for ACT is the measured $x$ - or gamma-ray attenuation of a source external to the waste drum. The ACT technology yields a quantitative attenuation map (related to density and atomic number) of the waste matrix within the drum. However, PCT can be used to measure and determine both the identity and the strength of any radioisotope source. The ray sum for passive or single-photon-emitted CT (sometimes called SPECT) imaging is the activity measured in disintegrations (d) per unit volume per unit time (e.g., Curies $=3.7 \times 10^{10}$ $\mathrm{d} / \mathrm{s}$ ) of the passive source within the waste drum. Therefore, a single-photon-emitted ray sum is the integrated radioisotope activity, modified by one or a multiple of exponential attenuations, along the path from the source position within the drum to the detector. The function that is imaged for PCT is the measured $\mathrm{x}$ - or gamma-ray activity at one or more energies of all detectable radioisotopes within a drum. The PCT technology localizes all detectable radioisotopes within the drum and accurately determines their identity using a spectroscopy detector and electronics. The spectrometry detection equipment collects the entire energy spectrum for each integration point and the radioisotopes are identified by their signature (characteristic peaks) within the energy spectrum.

\section{Progress}

We continued to characterize the performance of IMPACT (Isotope Measurement by Passive and Active Computed Tomography) by measuring well characterized drums. One of the drums was a SGS calibration drum with approximately 2 grams of plutonium distributed over more than a third of the length of the drum. We also used our "mock waste" drum with calibrated sources of plutonium in different regions of the drum. The sources all had different isotopic distributions and had a combined mass of 1.5 grams of

${ }^{239} \mathrm{Pu}$. This drum allows us to study various levels of Pu loading in three very different matrices. We were able to achieve good agreement with the expected assay values for these drums if we used long data acquisition times. The need for these long times led to our redesign of LMPACT that will enable us to do a better job of certification with more reasonable measurement times.

We also used IMPACT to scan calibrated point sources of high specific activity with simple uniform attenuators. These tests were used to verify the performance of the new 
developments in our image reconstruction code. These data also helped us to investigate the effect of counting statistics and background in our assay error analysis.

We modified the IMPACT system to a new geometry that increases our counting efficiency. The collimator geometry of the new design looks more like an operating system instead of a research tool. Therefore the test data from this system will be better able to certify the single-detector A\&PCT scanner concept. In addition to modifying our system geometry we also made several changes in our data acquisition software to reduce the overhead time in our measurements.

Our reconstruction algorithm work over the past year has been significant. We have improved the 3D code developed in collaboration with UCSF in many ways. Because many different image reconstruction options were required, it became much simpler to separate the simulator from the reconstructor. Hence the two are now individual standalone codes. The reconstructor was upgraded to convert its final result from total measured disintegrations to an absolute assay based on the detector parameters. We also implemented constraints to bound the solution to zero in areas outside of the feasible reconstruction volume, such as outside the drum. We incorporated a sum-of-squares and chi-square error estimate to determine the accuracy of the fit between the measured sinogram data and the sinograms generated by the algorithm based on the current image estimate. This allowed us to better track the convergence of the algorithm. We implemented the ability to use a measured collimator response function instead of one calculated from the collimator dimensions. This was required to reconstruct data measured with systems using septa to improve the collimation aspect ratio. We upgraded the code to compensate for a measured background level. And, finally, for experimental purposes, we created a version of the code which incorporates a background estimation scheme developed at LANL.[PRE94]

We have also undertaken to entirely rewrite the reconstruction software based on the physics and geometry of the drum imaging problem. Our current code, which was developed in collaboration with UCSF, was adapted from a code specifically designed for medical imaging geometries, which have much larger collimator aspect ratios. There were several assumptions in the code which were valid for the medical imaging case, but not for the drum imaging problem. The new code, which is nearly complete, will be more accurate for our imaging environment, should be faster than the current code, and will be more flexible. The increased flexibility will give us the capability to characterize the problem as a matrix inversion, which allows us to use additional algorithms such as SVD and constrained conjugate gradient methods to aid in the solution.

We have also improved the gamma-ray spectroscopy analysis of our data. Our HPGe spectrometer has been fully characterized for the GRPANL and GAMANAL analysis codes. We have developed GRPANL routines to analyze high statistics spectra to extract peak areas of gamma-ray lines for isotopic evaluation. We have also begun to survey the various "self-attenuation corrections methods to determine which would be the most applicable to our data.

\section{Technical Description}

The A\&PCT scanner at LLNL is shown in Figure 1 and is called IMPACT (Isotope Measurements by Passive and Active Computed Tomography). The IMPACT system is 
capable of scanning full-sized TRU waste drums that weigh up to 1000 pounds. The IMPACT scanner consists of two towers constructed of aluminum interlocking spaceframe tubing. One tower supports the active $166 \mathrm{~m}_{\mathrm{Ho}}$ isotope source and the other supports a single high-purity Germanium (HPGe) detector system. The towers are designed to be versatile so that additional sources and detectors could be added to the scanner in the future. Between the two supporting towers is a three-axis drum manipulator.

The manipulator is capable of translating and elevating a 1000 pound waste drum 50 inches. In addition, the manipulator rotates the drum 360 degrees. The drum manipulator was designed to be robust with safety features that are necessary for handling TRU waste in a seismic active area. Engineering safety notes were developed for the entire IMPACT scanning system.

A one-ton Jib crane is located at one end of the IMPACT scanner and provides easy and safe loading of the heavy waste drums (see Fig. 2). The crane is interlocked in a home position away from the drum manipulator to assure that the waste drum cannot be driven into the jib crane during system operation.

The IMPACT scanner uses a PC for system control and acquisition. During both active and passive operations, the PC control computer positions the drum for data acquisition. After positioning, the control computer communicates with a multi-channel analyzer (MCA) that acquires data from the HPGe energy-discriminating detector. The detector integration time is variable, and when completed, the control computer downloads the MCA's data and stores selected energy regions of interest and/or the entire spectrum onto a system disk. After the data is stored, the control computer and manipulator move the drum to the next integration position (also known as ray sums). The PC again acquires data from the MCA and HPGe detector system. The IMPACT scanner translates the drum in 2-inch increments after each integration point. A 208-L drum is translated 14 times and then rotated approximately 17 degrees. Each set of 14 ray sums make up a projection. After the rotation, the next projection of 14 integration points are acquired with a 2 -inch translation after each one. This process is repeated for 21 rotations (or projections) before the drum is elevated 2 inches and the process is repeated for the next level or slice plane. IMPACT acquires 18 slice planes for a $208-\mathrm{L}$ drum.

The data acquired from IMPACT is reconstructed and analyzed on a UNIX based SGI or SUN work station, not the PC. A UNIX file system is mounted by the PC and data are transferred over an ethernet cable. Both the energy regions of interest (EROI) and/or spectra that represents each individual ray sum are transferred. The advantage of this method is that the data can be analyzed on the UNIX system as it is being acquired.

\section{Photographic Description}

A photograph of the IMPACT scanner is shown if Fig. 1. The drum loading method is shown in Fig.2. 


\section{Experimental Results}

We have characterized the performance of IMPACT (Isotope Measurement by Passive and Active Computed Tomography) by measuring well characterized drums. One of the drums was a SGS calibration drum with approximately 2 grams of plutonium distributed over more than a third of the length of the drum. We also used a "mock waste" drum with calibrated sources of plutonium in different regions of the drum. The sources all had different isotopic distributions and had a combined mass of 1.5 grams of ${ }^{239} \mathrm{Pu}$. This drum allows us to study various levels of Pu loading in three very different matrices. We were able to achieve good agreement with the expected assay values for these drums if we used long data acquisition times. The need for these long times led to our redesign of IMPACT that will enable us to do a better job of certification with more reasonable measurement times.

We also used IMPACT to scan calibrated point sources of high specific activity with simple uniform attenuators. These tests were used to verify the performance of the new developments in our image reconstruction code. These data also helped us to investigate the effect of counting statistics and background in our assay error analysis.

\section{Performance}

We have measured the performance of the A\&PCT system using the controlled experiments described in the previous section. The results on the intense radioactive sources show that the A\&PCT technology can determine the activity with an accuracy of better than $10 \%$. For the weaker sources with more complicated attenuation matrices we again found good agreement with the known values (10-15\%) but the performance is strongly limited by the statistics of the data sets.

\section{Waste Performance}

The A\&PCT technology has also been used to measure real TRU waste drums. This has been performed by the IMPACT system at LLNL as well as the system in the BIR/WIT trailer (see commercialization section below). The results of the A\&PCT assays generally agreed with the listed values in those cases where the waste matrix and source distribution was favorable for SGS or neutron assay techniques. Serious disagreements were found for strongly attenuating matrices and for non homogeneous distributions of the radioactive material. More conclusive testing of the A\&PCT technology could be done using mock waste matrices with well characterized radioactive sources.

A summary of the preliminary performance of our A\&PCT systems (both LLNL's IMPACT and BIR's WIT-A\&PCT) is given in Table 1. Table 1 is in the format of the Waste Isolation Pilot Plant Waste Acceptance Criteria TRU Waste Characterization Quality Assurance Program Plan.[QAPP] This is also the table used in the Performance Demonstration Plan for Nondestructive Assay for the TRU Waste Characterization Program.[PDP95] The results show our prliminary performance obtained from a few calibration waste drums as a function of waste activity. The assay results are cbased on 
the analysis of the ${ }^{239} \mathrm{Pu} 414-\mathrm{kev}$ gamma-ray peak. No self attenuation (lump) corrections were made for these data.

\section{Design Considerations}

Perhaps the most important design consideration for this technology is the system throughput. In the current scan protocol each drum slice requires almost 300 separate measurements for both the active and passive scans. For a typical drum we need to measure 10 or more slices. Combining this with a desired measurement time of about 10 seconds per view results in a throughput of about 1 drum per day. This could be greatly increased by improving our reconstruction algorithms to deal with fewer data points in the characterization of the drums. The throughput could also be enhanced by incorporating several high-purity germanium detectors into the system. We are currently exploring a new design that would use 5 detectors that would yield a far better throughput.

\section{PUBLIC INVOLVEMENT RECORD}

\section{Hazards}

The A\&PCT system we propose requires the use of radioactive sources. There are regulatory requirements associated with the use of a radioactive source, but these requirements are and have been easily met by both the national laboratories and private industrial users for many years. This regulatory guideline is not significant. We have already separately transferred the active ${ }^{166 \mathrm{~m}} \mathrm{Ho}$ source from LLNL to BIR and back, it will not be carried on the mobile WIT system.

\section{Safety Analysis}

A general safety analysis for the A\&PCT technology has not been performed. However we generated operating safety procedures specific to LLNL that allow us to do measurements on TRU-waste drums in our facility. In addition the A\&PCT technology has been described in the Safety Analysis Document for the BIR/WIT trailer. This document was the basis for the operation of the trailer at LLNL, Rocky Flats Environmental Technology Site, and the Radioactive Waste Management Complex (RWMC) at the Idaho National Engineering Laboratory (INEL).

\section{END-USER SPONSORSHIP RECORD}

\section{Patents/Commercialization}

In FY1993, we began a technology transfer effort with an industrial partner, Biolmaging Research Inc. (BIR) of Lincolnshire, IL. They received funding in July 1993, from DOE Morgantown to develop a mobile Waste Inspection Tomography (WIT) system. In FY96, BIR plans to DT\&E this newly developed WIT system at several DOE sites. As a part of their mobile WIT system, they have installed a single, HPGe detector with a drum platform able to carryout tomographic scans on waste drums. In FY95, we have 
transferred and implemented revision 1.0 of our A\&PCT data acquisition and image reconstruction codes. Newer revisions of these codes and revisions of our customdeveloped, gamma-ray spectrum fitting and isotopic identification codes developed for waste assay were delivered in early FY96. This mobile system has been demonstrated and tested at LLNL in December 1995 through February 1996. INEL's RWMC is another field demonstration site for LLNL's A\&PCT technology. Since BIR is a private company, whatever requirements there are for the transfer of the A\&PCT technology to the commercial sector, they will become clear to BIR, and to DOE, by the time the initial DT\&E field trials are completed. The LLNL WIT funded effort is to transfer and implement the A\&PCT technology with the BIR technologies. This funding is not used to research and develop the A\&PCT technology. Therefore, this effort is fundamental to providing private industry with new technologies.

References

[AND91] B. C. Anderson and D. J. Osetek, Nondestructive Examination and Assay System Research and Development Requirements to Meet Evolving Regulations for Transuranic and Low-Level Wastes, draft report, Waste Isolation Pilot Plant, Carlsbad, NM (1991).

[MAR92] H. E. Martz, G. P. Roberson, C. Robert-Coutant, D. J. Schneberk, and D. C. Camp, Quantitative Waste-Form Assay Using Gamma-ray Computed Tomography, Lawrence Livermore National Laboratory, Livermore, CA, UCRL-JC-1 10648 (1992); Proc. Int. Symp. Spectrosc. and Struct. Molec. Nuclei, March 27-29, 1992, Tallahassee, FL.

[MAR95] "Radioactive Waste Realities as Revealed X- and Gamma-Ray Measurements," H. E. Martz, D. J. Decman, G. P. Roberson and D. C. Camp, Lawrence Livermore National Laboratory, Livermore, CA and Richard T. Bernardi, Biolmaging Reseach, Inc., Lincolnshire, IL, American Chemical Society Special Symposium on Emerging Technologies in Hazardous Waste Management VII, Atlanta, GA, September 1995.

[PDP95] "Performance Demonstration Program Plan for Nondestructive Assay for the TRU Waste Characterization Program," U.S. Department of Energy, Carlsbad Area Office, National TRU Program Office, CAO-94-1045, Revision 0, March 1995.

[PRE94] "A Maximum-Likelihood Reconstruction Algorithm for Tomographic Gamma-Ray Nondestructive Assay," T. H. Prettyman, R. A. Cole, R. J. Estep, and G. A. Sheppard, Los Alamos National Laboratory Report LA-UR94-1650.

[QAPP] "TRU Waste Quality Assurance Program Plan," U.S. Department of Energy, Carlsbad Area Office, National TRU Program Office 1994. 
[ROB94] "Characterization of Waste Drums Using Nonintrusive Active and Passive Computed Tomography," G. Patrick Roberson, Harry E. Martz, Daniel J. Decman, David C. Camp, Stephen G. Azevedo, and Eric R. Keto, Proceedings of the Nondestructive Assay and Nondestructive Examination Waste Characterization Conference, Pocatello, Idaho, February 14-16, 1994.

[WES94] Bill Weston et al., DOE NDANDE Waste Characterization Instrumentation Data Base, document in preparation by NDA/NDE Interface Working Group, Waste Isolation Pilot Plant, Waste Isolation Division, Westinghouse, Carlsbad NM (1994).

\section{List of Participants}

\section{Lawrence Livermore National Laboratory:}

Dr. Harry E. Martz, Jr., Nuclear Chemist, Principal Investigator, 1994 -

Dr. Daniel J. Decman, Nuclear Chemist, Co-Principal Investigator

Mr. G. Pat Roberson, Lead Engineer

Mr. Erik Johansson, Electrical Engineer

Dr. David C. Camp, Physicist, Consultant

BioImaging Research, Inc.

Richard Bernardi

Dave Entwistle

\section{UCSF}

Bruce Hasegawa

Keenan Brown 
Table 1 Summary of the preliminary performance characteristics of A\&PCT.

\begin{tabular}{|c|c|c|c|c|}
\hline $\begin{array}{c}\text { Range of } \\
\text { Waste Activity } \\
\text { ( } \alpha \text {-Curies) }\end{array}$ & $\begin{array}{c}\text { Nominal } \\
\text { Compliance } \\
\text { Point } \\
\alpha-\text { Curies } \\
\text { (g WG Pu) }\end{array}$ & $\begin{array}{c}\text { Relative } \\
\text { Precision } \\
\left(\mathbf{R}_{\mathrm{p}}\right)\end{array}$ & $\begin{array}{c}\text { Instrument } \\
\text { bias } \\
(\% \mathbf{R})\end{array}$ & $\begin{array}{c}\text { Total } \\
\text { Accuracy } \\
(\% \mathbf{R})\end{array}$ \\
\hline $\begin{array}{c}>0-0.04 \\
\text { LLNL mock } \\
\text { w/ PIDIE }\end{array}$ & $\begin{array}{c}0.008 \\
(0.1) \\
(0.150-0.200)\end{array}$ & $\begin{array}{l}40 \\
-\end{array}$ & $\begin{array}{c}75-125(25) \\
90-110(10 \%)\end{array}$ & $\begin{array}{c}\text { Low 40\% } \\
\text { High 175\% } \\
-\end{array}$ \\
\hline $\begin{array}{c}>0.04-0.4 \\
\text { LLNL mock } \\
\text { w/ PIDIE }\end{array}$ & $\begin{array}{c}0.08 \\
(\mathbf{1 . 0}) \\
(\mathbf{0 . 7 7 5 )}\end{array}$ & $\begin{array}{r}30 \\
-\end{array}$ & $\begin{array}{c}50-150(50) \\
90-110(<10 \%)\end{array}$ & $\begin{array}{c}\text { Low 30\% } \\
\text { High 200\% } \\
-\end{array}$ \\
\hline $\begin{array}{c}>0.4-4.0 \\
\text { SGS } \\
\text { calibration }\end{array}$ & $\begin{array}{c}\mathbf{0 . 8} \\
(\mathbf{1 0 )} \\
(\mathbf{1 9 . 6})\end{array}$ & $\begin{array}{r}20 \\
-\end{array}$ & $\begin{array}{c}50-150(50) \\
65-135(35 \%)\end{array}$ & $\begin{array}{c}\text { Low 30\% } \\
\text { High 200\% } \\
-\end{array}$ \\
\hline$>4.0$ & $\begin{array}{c}12.8 \\
(160)\end{array}$ & 10 & $\begin{array}{c}75-125(25) \\
- \\
\end{array}$ & $\begin{array}{c}\text { Low 50\% } \\
\text { High 150\% }\end{array}$ \\
\hline
\end{tabular}



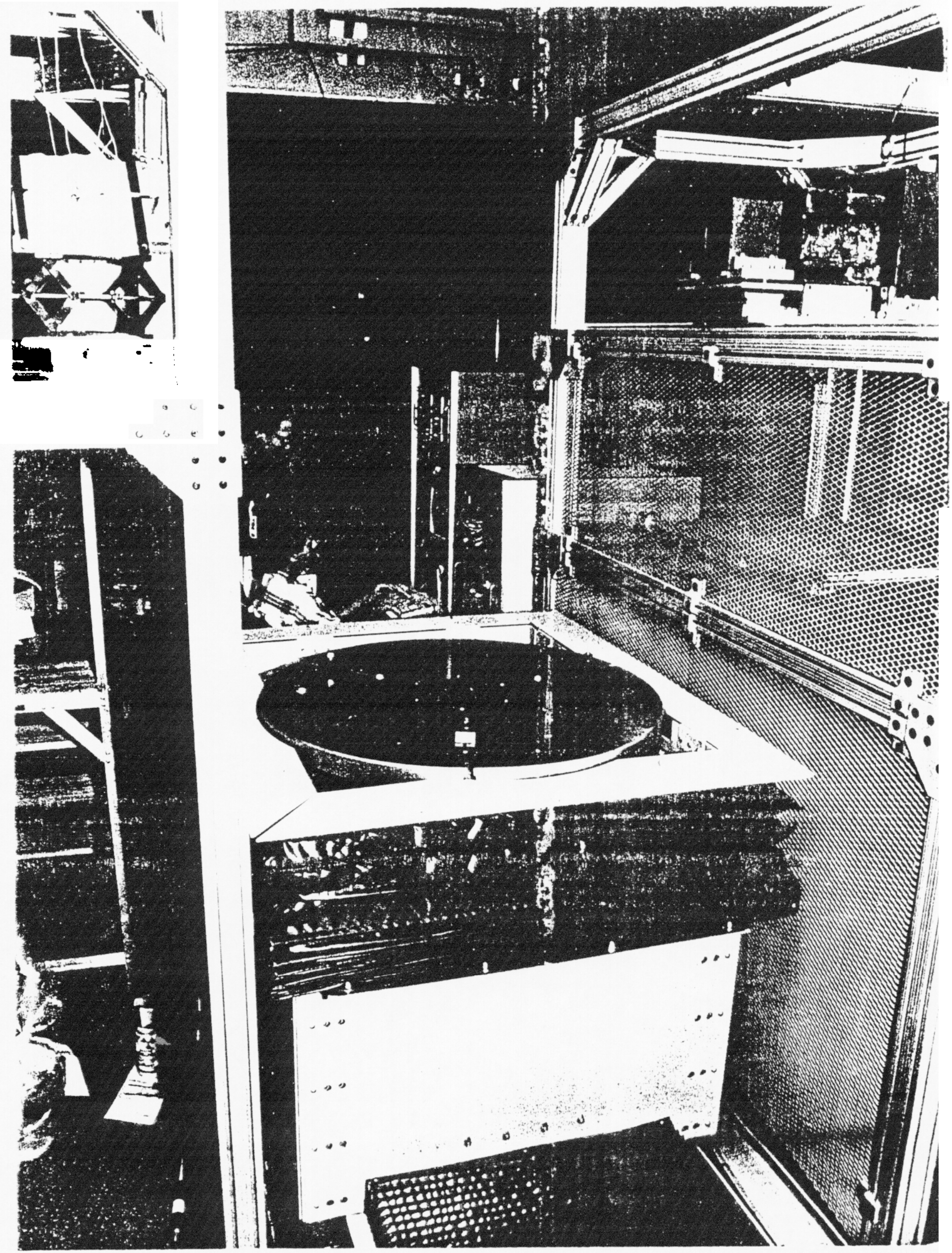


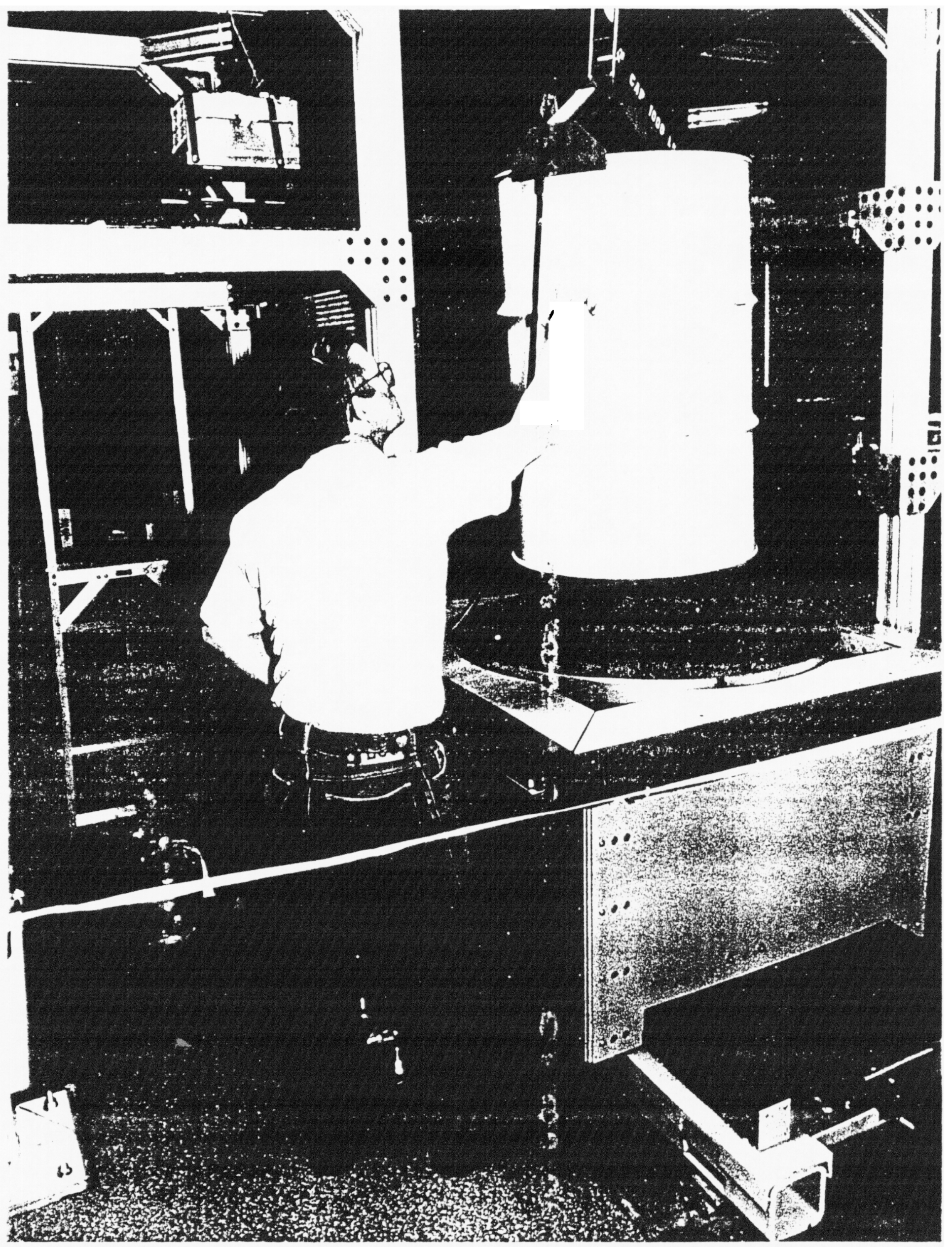




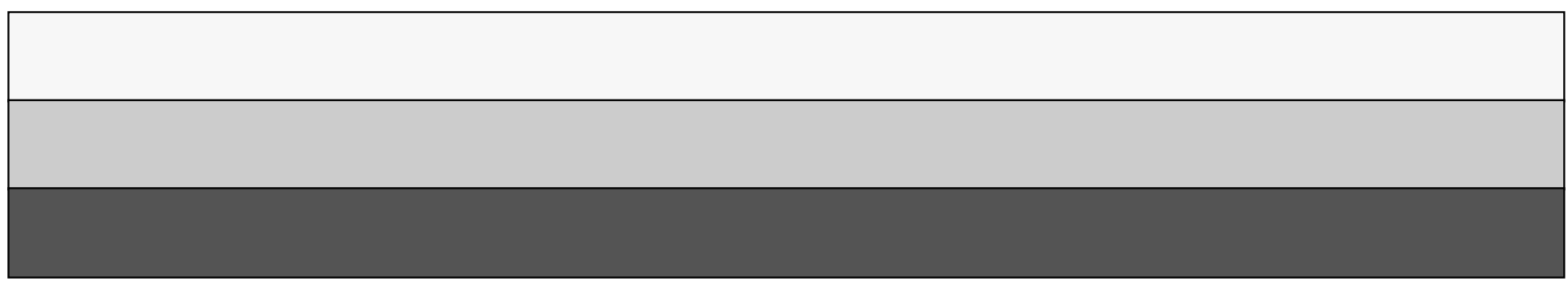

